\title{
Fuzzy Reliability Design on Mechanical Components under Normal Distribution Based on Entropy Theory
}

\author{
Li Zhong, Bo Yucheng \\ (North University of China, Taiyuan, China, 030051)
}

\begin{abstract}
In fuzzy reliability design on Mechanical Components, the determination of characteristic value of subordinate function mainly depends on the experience that accumulated and summarized by researchers. Thus, it has strong subjectivity. In this paper, through entropy theory the relationship between determining characteristic value of subordinate function and reliability made a great description. Finally, the examples prove this method's feasibility.
\end{abstract}

Keywords-fuzzy theory; entropy theory; normal distribution; reliability

\section{INTRODUCTION}

In the reliability design of mechanical components, two kinds of design schemes have been adopted mainly: 1) the stress and strength of material is assumed to be constant, and then according to certain strength conditions designed or tested. In this method, all the parameters and formula are obtained mostly by experience. Thus, based on these results it is difficult to make reasonable design; 2) since various variables and their corresponding stresses, strengths of materials are not definite, the fuzzy reliability theory is carried in the design. In view of fuzziness, different methods are used to determine membership function. But determination of membership function is based on summarization of long-standing practical experience and study of spare components' use, and experts' evaluation. Therefore, it has strong subjectivity and empirical, making design not very accurate. Entropy theory used in this paper makes a very great description of the relationship between function characteristic value and reliability design. Take the normal distribution of the machine components of fuzzy reliability design as an example, the paper shows the method in fuzzy reliability design is feasible.

\section{FUZZY PROBABILITY AND FUZZY ENTROPY DISCRETE}

Fuzzy event probability is

$$
\mathrm{P}_{\mathrm{D}}(\mathrm{Z})=\sum_{\mathrm{i}=1}^{\mathrm{n}} \mathrm{u}_{\mathrm{z}}\left(\mathrm{x}_{\mathrm{i}}\right) p\left(\mathrm{x}_{\mathrm{i}}\right)
$$

in which $\mathrm{Z}$ is discrete fuzzy event; $p\left(\mathrm{x}_{\mathrm{i}}\right)$ is basic discrete event probability ; $u_{z}\left(x_{i}\right)$ is discrete fuzzy event membership function and $n$ is number of samples. Continuous fuzzy event probability is

$$
P_{C}(Z)=\int \mu_{Z}(x) f(x) d x
$$

in which $\mu_{Z}(x)$ is continuous fuzzy event membership function; $f(x)$ is probability density function of continuous random variables. The entropy of discrete event and the entropy of continuous events are as follows, respectively.

$$
\begin{array}{r}
H_{D}(Z)=\sum_{i=1}^{n} p\left(x_{i}\right) \log \left[p\left(x_{i}\right)\right] \\
H_{C}(Z)=-\int_{-\infty}^{+\infty} p(x) \log [p(x)] d x
\end{array}
$$

Combine (1), (2), (3) and (4), the entropies of discrete fuzzy events and continuous mode paste events for entropy can be drawn.

$$
\begin{aligned}
& H_{D}(Z)=-\sum_{i=1}^{n} \mu_{A}\left(x_{i}\right) p\left(x_{i}\right) \log \left[\mu_{A}\left(x_{i}\right) p\left(x_{i}\right)\right] \\
& H_{C}(Z)=-\int \mu_{A}(x) f(x) \ln \left\lfloor\int \mu_{A}(x) f(x)\right\rfloor d x \\
& H_{c}(Z) \text { is uncertainty of fuzzy event } Z \text {. }
\end{aligned}
$$

III. FUZZY RELIABILITY AND FUZZY ENTROPY OF MeChanical COMPONENTS IN FAILURE MODES TO OBEY NORMAL DISTRIBUTION

The normal distribution is one of the most common distribution forms in the mechanical reliability design. The past research has confirmed that the static stress and variable stress distribution model of the mechanical components belong to normal distribution, such as the wear life of mechanical components, and so on. The mechanical components during working process are under cyclic loading, which causes mechanical components to have various damage degrees.

In the fuzzy reliability design, the randomness of stress and strength should be considered. According to Literature, the components' strength $r$ and stress $s$ are considered to random variables obeying normal distribution. Their means are $\mu_{\mathrm{r}}$ and $\mu_{\mathrm{s}}$, standard deviations $\sigma_{\mathrm{s}}$ and $\sigma_{\mathrm{r}}$, and probability density function $f_{r}(r)$ and $f_{s}(s)$, respectively.

General normal distribution probability density function formula is

$$
f(x)=(2 \pi)^{\frac{1}{2}} S^{-1} \exp \left[\frac{(x-\bar{\sigma})^{2}}{2 S^{2}}\right]
$$

Where $\bar{\sigma}$ is stress mean and S stress standard deviation. 
Interference random variable $t=\bar{t}-\bar{s}$ is random variables of normal distribution. The mean is $t=\mu_{t}-\mu_{s}$ and standard deviation $\sigma=\sqrt{\sigma_{r}^{2}+\sigma_{s}^{2}}$.

When the stress and intensity distribution are in accordance with normal distribution, reliability can be signified as

$$
\begin{aligned}
R & =\int_{0}^{-\infty} f(t) d t=\int_{0}^{-\infty} \frac{1}{\delta_{t} \sqrt{2 \pi}} \exp \left[-\frac{\left(t-\mu_{t}\right)^{2}}{2 \sigma_{t}^{2}}\right] d t \\
& =\varphi\left(t_{R}\right)
\end{aligned}
$$

In which $\varphi\left(t_{R}\right)$ is standard normal distribution function value of coupling coefficient $t_{R}$ andis coupling equation of normal distribution. $\mu(\mathrm{t})$ is used to describe fuzzy variables, $\mathrm{S}$ security state space, $\mathrm{Q}$ failure state space of fuzzy variables and the relationship between $\mathrm{S}$ and $\mathrm{Q}$ is $\mathrm{S}+\mathrm{Q}=1$. The fuzzy variable function has several different types, such as trapezoidal distribution, normal distribution, cauchy distribution, etc.. The stress of mechanical components ranges from permitted usage stage to forbidden usage stage.

This stage is based on membership functions of normal distribution.

The membership functions for the security state space and failure state space functions are shown in formulas (9) and (10),

$$
\begin{aligned}
& \mu_{s}(t)=\left\{\begin{array}{c}
\exp \left[-(t-d)^{2} / \alpha\right] \\
1
\end{array}\right\} \begin{array}{l}
t \leq d \\
t>d
\end{array} \\
& \mu_{Q}(t)=1-u_{s}(t)=\left\{\begin{array}{c}
1-\exp \left[-(t-d)^{2} / \alpha\right] \\
0
\end{array}\right\} \begin{array}{l}
t \leq d \\
t>d
\end{array}
\end{aligned}
$$

in which and $\alpha$ are mean and variance of membership function. In the following calculation of fuzzy reliability and fuzzy failure probability, it can be displayed that characteristic values of membership values are determined. However, its determination is influenced by researcher's subjectivity, causing inaccurate calculation. Therefore, entropy theory is introduced into the research, where the set value range of $\mathrm{d}$ and can be made quantity description. Formulas (9) and (10) are substituted for (2), the fuzzy reliability of mechanical components can be drawn as follows,

$$
\begin{aligned}
& R_{s}=\int_{-\infty}^{+\infty} \mu_{s}(t) f(t) d t \\
& =\int_{-\infty}^{d} \exp \left[-(t-d)^{2} / \alpha\right] \frac{1}{\sigma_{t} \sqrt{2 \pi}} \exp \left[-\frac{\left(t-\mu_{t}\right)^{2}}{2 \sigma_{t}^{2}}\right] d t \\
& +\int_{d}^{+\infty} \frac{1}{\sigma_{t} \sqrt{2 \pi}} \exp \left[-\frac{\left(t-\mu_{t}\right)^{2}}{2 \sigma_{t}^{2}}\right] d t
\end{aligned}
$$

The fuzzy failure probability is

$$
\begin{gathered}
R_{Q}=\int_{-\infty}^{+\infty} \mu_{Q}(t) f(t) d t= \\
\int_{-\infty}^{d}\left\{1-\exp \left[-(t-d)^{2} / \alpha\right]\right\} \frac{1}{\sqrt{2 \pi}} \sigma_{t} \exp \left(-\frac{t-\mu_{t}}{2 \sigma_{t}{ }^{2}}\right) d t
\end{gathered}
$$

Formulas (9) and (10) are substituted for (6), the uncertainty of the fuzzy reliability is gotten

$$
\begin{aligned}
& H_{Z}(Z)=-\int_{-\infty}^{+\infty} \mu_{s}(t) f(t) \ln \left[\mu_{s}(t) f(t) d t\right] \\
&=-\int_{-\infty}^{d} \exp \left[-(t-d)^{2} / \alpha\right] \frac{1}{\sigma_{t} \sqrt{2 \pi}} \exp \left(-\frac{t-\mu_{t}}{2 \sigma_{t}^{2}}\right) \times \\
& \ln \left\{\exp \left[-(t-d)^{2} / \alpha\right] \frac{1}{\sigma_{t} \sqrt{2 \pi}} \exp \left(-\frac{t-\mu_{t}}{2 \sigma_{t}^{2}}\right)\right\} d t- \\
& \int_{d}^{+\infty} \frac{1}{\sigma_{t} \sqrt{2 \pi}} \exp \left[-\frac{\left(t-\mu_{t}\right)^{2}}{2 \sigma_{t}^{2}}\right] \ln \left\{\frac{1}{\sigma_{t} \sqrt{2 \pi}} \exp \left[-\frac{\left(t-\mu_{t}\right)^{2}}{2 \sigma_{t}^{2}}\right]\right\} d
\end{aligned}
$$

(13)

The entropy of the uncertainty of the fuzzy reliability is

$$
\begin{gathered}
H_{Q}(Z)=-\int_{-\infty}^{+\infty} \mu_{Q}(t) f(t) \ln \left[\mu_{Q}(t) f(t) d t\right]= \\
-\int_{-\infty}^{d}\left\{1-\exp \left[-(t-d)^{2} / \alpha\right]\right\} \frac{1}{\sqrt{2 \pi}} \sigma_{t} \exp \left(-\frac{t-\mu_{t}}{2 \sigma_{t}^{2}}\right) \times \\
\ln \left\{\left\{1-\exp \left[-(t-d)^{2} / \alpha\right]\right\} \frac{1}{\sqrt{2 \pi}} \sigma_{t} \exp \left(-\frac{t-\mu_{t}}{2 \sigma_{t}^{2}}\right)\right\} d t
\end{gathered}
$$

Based on Formulas (13) and (14), according to the characteristic value $d$ and $\alpha$ under acceptable degree in the initial assumptions, the fuzzy reliability can be gotten by calculation.

\section{CASE ANALysis}

During work process the machine workpiece will be influenced by alternating stress. Its strength and stress are confined to normal distribution. The stress mean is, $\bar{S}=80 M P a$.

the strength mean is $\bar{r}=120 \mathrm{MPa}$ and the standard deviation is. $\sigma_{r}=15 \mathrm{MPa}$ Stress intensity is assumed as fuzzy random variable, try to solve the machine workpiece's entropy of fuzzy reliability degree and fuzzy reliability uncertainty degree.

The analysis indicates that the strength and stress obey normal distribution. According to the nature of normal distribution, $\mathrm{Z}$ and $\sigma_{Z}$ can be solved. 
(1) The interference random variable mean, gotten by $\bar{r}-\bar{s}$, is $\bar{Z}=40$

(2)The interference random variable standard deviation, gotten by $\sqrt{\sigma_{t}^{2}+\sigma_{s}^{2}}$ is. $\sigma_{z}=25$

(3)The reliability degree, gotten by formulas (8), is $R_{z}=\int_{0}^{+\infty} f(z) d z=\int_{0}^{+\infty} \frac{1}{\sigma_{t} \sqrt{2 \pi}}\left[\frac{\left(z-\mu_{z}\right)^{2}}{2 \sigma_{z}{ }^{2}}\right] d z=0.9452$

(4) Suppose that $\alpha=\alpha_{0} \delta_{z}, d=d_{0} \mu_{z}$ and at random set $\alpha_{0}=1,3,6 ; d_{0}=1$ then $\alpha=25,75,150 ;$ When $d=0 \sim \mu_{z}$, The change curve of $\mathrm{R}_{\mathrm{Z}}$ and $\mathrm{H}_{\mathrm{Z}}$ with $d$ change is obtained shown in Fig 1 and Fig 2.

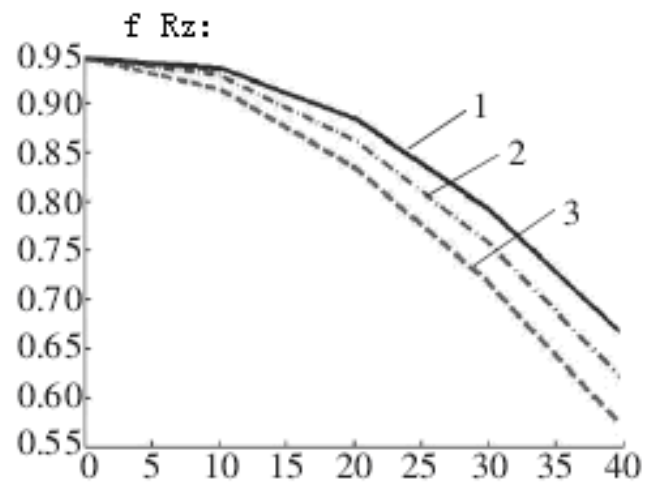

Fig 1. a-d-Rz Curve

1. $a=150$ 2. $a=75$ 3. $a=25$

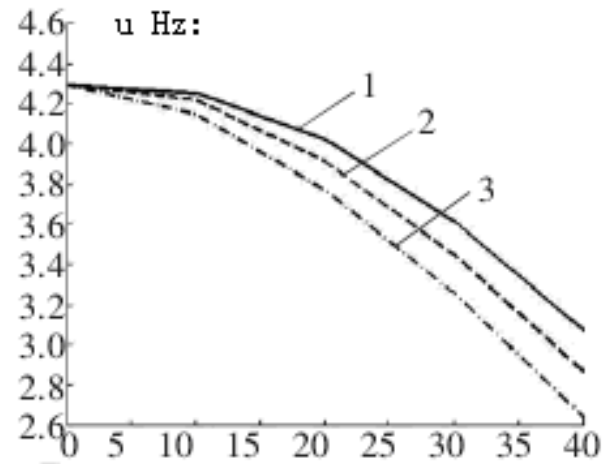

Fig 2. a-d-Hz Curve 1. $a=150$ 2. $a=75$ 3. $a=25$

As $\quad \alpha_{0}=1, d_{0}=1,3,5 ; \alpha_{0}=25, d_{0}=40,120,200$. The curve of $\mathrm{R}_{\mathrm{Z}}$ and $\mathrm{H}_{\mathrm{Z}}$ with $d$ change is obtained. The change trend of fuzzy reliability $\mathrm{R}_{\mathrm{Z}}$ and uncertainty degree can be shown, that is, they decrease as $d$ increases. As a $=25$ and $\mathrm{d}=5, \mathrm{R}_{\mathrm{Z}}=0.9018$ and $\mathrm{H}_{\mathrm{Z}}=4.0855$. Thus, the reasonable characteristic values can be chosen from them.

\section{CONCLUSION}

It is feasible that fuzzy function characteristic values are selected by entropy theory, which provides reference for fuzzy reliability design. According to the test the method in fuzzy reliability design is feasible.

\section{REFERENCES}

[1] JI yu-jie. Mechanism Reliability Simulation Technology Study[M]. Shenyang: Northeast University, 2006.

[2] LI Yang-xing,LI Guang-yu. Fuzzy reliability design of gear strength based on entropy theory[J]. Journal of Machine Design, 2004(2): 3839.

[3] LIANG Qing-wei, SONG Bao-wei, MAO Zhao-yong. Reliability Fuzzy Distribution Method based on Entropy Weight[J]. Fire Control \& Command Control, 2005(7): 73-76.

[4] MO Caisong, QIAN Xueming. Analysis on the Fuzzy Reliability Design of Machine Components Strength[J]. Machinery Design \& Manufacture, 2007(4):33-34.

[5] MOU Zhi-zhong. Reliability Design of Machenicas Parts[M]. Beijing: Machinery Industry Publishing House, 1998.

[6] WANG Zheng-lin, LIU Ming. Proficient in Matlab7[M]. Beijing: electronics industry Publishing house, 2006.

[7] ZHANG Wen-xiu, Liang Yi. On Uncertainty Reasoning[M]. Xi'an: Journal of Xi'an Jiaotong University Publishing House, 1996. 